\title{
Algoritmo LMS Restrito Aplicado ao Problema de Otimização de um Conformador de Feixe de Uplink-Downlink via Dualidade
}

\author{
Ciro A. Pitz, Marcelo G. Vanti, Orlando J. Tobias
}

\begin{abstract}
Resumo-Este trabalho apresenta um algoritmo para um ótimo Conformador de Feixe para os enlaces de subida e descida (uplink-downlink) em uma Estação de Rádio Base (ERB), que procura iterativamente atender determinado valor de Razão Sinal-Interferência mais Ruído (SINR), tanto no móvel quanto na ERB. A relação entre os dois canais é obtida através da Teoria da Dualidade. O atual algoritmo foi originalmente proposto por Viotsky e Madhow, aqui modificado com a introdução do algoritmo LMS restrito para a obtenção dos pesos ótimos do enlace de subida (uplink). Tal método mostra ser eficiente, e é comprovado em simulações numéricas presentes no final do trabalho.
\end{abstract}

Palavras-chave-Arranjo de Antenas, Algoritmos Adaptativos com Restrições, Rejeição de Interferência e Ruído em Sistema de Comunicação Celular.

Abstract- This paper presents an algorithm for an optimum uplink-downlink beamformer in a Radio Base Station (RBS), which iteratively search to attend determined value of Signal to Interference plus Noise Ratio (SINR) in both mobile and in the RBS. The relationship between the two channels is obtained by the Duality Theory. The current algorithm was originally proposed by Viotsky and Madhow, which then was complemented in this work by the constrained LMS algorithm to obtain the optimal weights for the uplink. This method shows to be efficient and is proven through numerical simulations in the end of this paper.

Keywords-Antenna Array, Adaptive Algorithms with Restrictions, Interference plus Noise Rejection in Cellular Systems.

\section{INTRODUÇÃO}

A teoria de dualidade tem sido aplicada com sucesso em diversas áreas de processamento de sinais, como por exemplo, em algoritmos com restrições para arranjo de antenas. No projeto de arranjos adaptativos de antenas, métodos de otimização são utilizados para a determinação dos valores ótimos dos pesos, realizando a conformação de feixe, e seus valores são sujeitos a determinadas restrições; como por exemplo, a especificação de valores mínimos para a SINR. A

Ciro A. Pitz, Marcelo G. Vanti e Orlando J. Tobias, LAP - Laboratório de Antenas e Propagação, Depto. de Eng. Elétrica e Telecomunicações, Universidade Regional de Blumenau, Blumenau, SC, E-mail: ciropitz@gmail.com, vanti@furb.br, orlando@furb.br.

Marcelo G. Vanti e Orlando J. Tobias são também Professores do Departamento de Engenharia Elétrica e Telecomunicações da Universidade Regional de Blumenau (FURB), Blumenau, SC, Brasil. dualidade apresenta uma abordagem alternativa para este tipo de problema e uma nova estrutura para o processador adaptativo, reformulando o problema original (primal) no espaço dual dos multiplicadores de Lagrange [1,2].

Os estudos em antenas adaptativas são realizados atualmente visando o sistema de comunicação celular, onde o principal objetivo é aumentar a eficiência espectral e a Qualidade de Serviço (QoS). O primeiro passo para o uso de antenas adaptativas é a implementação na Estação de Rádio Base (ERB) de um algoritmo de conformação de feixe, que é realizada na recepção (canal de uplink) e na transmissão (canal de downlink) [3]. A principal tarefa do algoritmo é maximizar a $S I N R$ em ambos os canais, sujeito a algumas restrições apropriadas como, por exemplo, potência irradiada. Assim, a partir de avaliar a transmissão (downlink), podem ser obtidos os pesos ótimos do enlace de subida (uplink), no qual a vantagem do método consta em considerar a antena recebendo ao invés de transmitindo [4], tal fato é baseado na maior simplicidade de trabalhar com o canal de subida. Tal método, chamado de dualidade de downlink-uplink, é de grande importância, pois o problema de recepção (uplink) tem mais fácil resolução que o problema de transmissão (downlink) [4-6].

A dualidade de downlink-uplink foi primeiramente estabelecida com aplicação em sistemas MISO, em [7] e [8]. Ali é discutido que os pesos ótimos de conformação de feixe de transmissão do enlace de descida, que minimizam a soma de potência sob a restrição de um mínimo SINR por sinal, são os mesmos (diferenciados por um fator de escala) que os pesos de conformação de feixe do enlace de subida [5].

A teoria de dualidade também é aplicada com sucesso em sistemas MIMO (Multiple Input, Multiple Output), que é a evolução do sistema de múltipla entrada uma saída (MISO). A comparação entre tais sistemas é ilustrada na Fig. 1; também mostrando o caso do sistema SIMO (Single Input, Multiple Output).

O algoritmo discutido neste trabalho é fundamentado na Teoria da Dualidade, estabelecendo a relação entre os enlaces de subida e descida (downlink e uplink), originalmente proposto em [8], apresentando um problema de otimização não solucionada em [8]. Sendo assim, a proposta deste estudo é fornecer uma solução para este problema. Para tal, é proposta a utilização do algoritmo LMS restrito, visando 
complexidade computacional e suas boas características de estabilidade. Neste trabalho, o algoritmo é descrito partindo de um sistema MISO, facilitando a formulação matemática quando comparado com um sistema MIMO. O algoritmo LMS restrito é implementado no método proposto em [8], e, assim, obtido um processo adaptativo que automaticamente procura atender simultaneamente a SIRN exigida na ERB e no móvel.

O trabalho está organizado da seguinte maneira: Na Seção II é apresentado o modelo do sinal do enlace de subida e descida. Na Seção III é derivada a relação entre os dois enlaces através da teoria da dualidade. A Seção IV apresenta o algoritmo proposto. Na Seção V, são apresentadas simulações numéricas para avaliar os resultados do algoritmo proposto. Finalmente, conclusões de este trabalho são dadas na Seção VI.

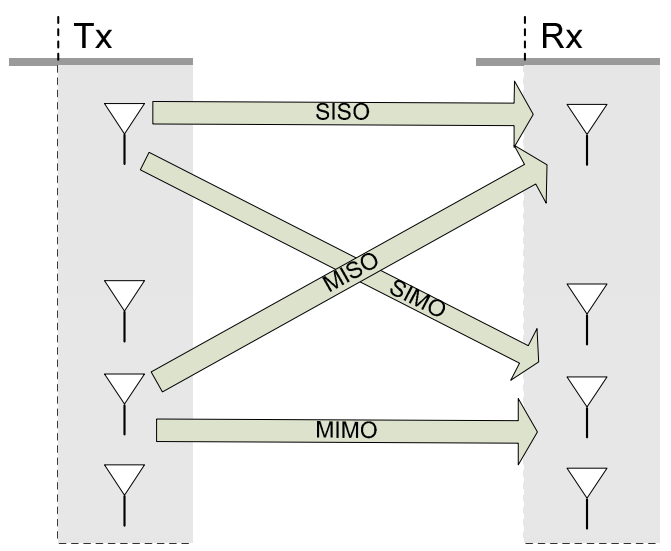

Fig. 1 - Sistemas SISO, MISO, SIMO e MIMO.

\section{MODElo DO SinAL}

Considerando um sistema MISO com $k$ antenas na ERB e $M$ usuários, o sinal transmitido pode ser descrito como

$$
\mathbf{x}(t)=\sum_{j=1}^{M} \mathbf{w}_{j} u_{j}(t)
$$

onde $\mathbf{w}_{j}$ e $u_{j}(t)$ são o vetor pesos e o sinal de enviado para o j-esimo usuário, respectivamente. Sem perda de generalidade, definem se todas as fontes de sinal $u_{j}(t)$ com a mesma potência normalizada $E\left|u_{j}(t)\right|^{2}=1$. Assim, o sinal recebido por cada usuário do sistema é escrito como

$$
y_{i}(t)=\mathbf{s}_{i}^{H} \mathbf{x}(t)+n_{i}=\sum_{j=1}^{M} \mathbf{s}_{i}^{H} \mathbf{w}_{j} u_{j}(t)+n_{i},
$$

ou, colocando em evidencia o $i$-esimo termo,

$$
y_{i}(t)=\mathbf{s}_{i}^{H} \mathbf{w}_{i} u_{i}(t)+\sum_{j \neq i}^{M} \mathbf{s}_{i}^{H} \mathbf{w}_{j} u_{j}(t)+n_{i}
$$

De (3), observamos que o primeiro, segundo e terceiro termo, representam, respectivamente, o sinal de interesse, interferente e ruído. $\mathrm{O}$ vetor $\mathbf{s}_{i}$ representa o canal entre as $k$ antenas transmissoras e o usuário $i$. Definindo a variância de $n_{i}$ como sendo $\sigma^{2}$, uma expressão de $\operatorname{SINR}$ para o usuário $i$ pode ser expressa como

$$
\operatorname{SINR}_{i}=\frac{\left|\mathbf{s}_{i}^{H} \mathbf{w}_{i}\right|^{2}}{\sum_{j \neq i}^{M}\left|\mathbf{s}_{i}^{H} \mathbf{w}_{j}\right|^{2}+\sigma^{2}} .
$$

De forma semelhante, chega-se à expressão de SINR na ERB referente ao enlace de subida,

$$
\operatorname{SINR}_{i}=\frac{P_{i}\left|\hat{\mathbf{w}}_{i}^{H} \mathbf{s}_{i}\right|^{2}}{\sum_{j \neq i}^{M} P_{j}\left|\hat{\mathbf{w}}_{j}^{H} \mathbf{s}_{i}\right|^{2}+\sigma^{2}\left\|\hat{\mathbf{w}}_{i}\right\|^{2}},
$$

onde $\hat{\mathbf{w}}_{i}$ é o vetor pesos do enlace de subida (uplink) na ERB, referente ao móvel $i$, e $P_{i}$ é a potência irradiada pela antena do terminal móvel $i$. Então, é definido o problema de otimização para os enlaces de subida e descida (downlink e uplink), representado pelas Eqs. (6) e (7), respectivamente. Assim,

$$
\min _{\mathbf{w}_{i}}\left(\sum_{i=1}^{M}\left\|\mathbf{w}_{i}\right\|^{2}\right)
$$

sujeito a: $\operatorname{SINR}_{i} \geq \gamma_{i}$

e

$$
\min _{\hat{\mathbf{w}}_{i} \ldots \hat{\mathbf{w}}_{M}, P_{i} \ldots P_{M}}\left(\sum_{i=1}^{M} P_{i}\right)
$$

sujeito a: $\operatorname{SINR}_{i} \geq \gamma_{i}$ e $\hat{\mathbf{w}}_{i} \mathbf{s}_{i}=1$

\section{DuAlidade DA CONFORMAÇÃO DE FEIXE}

Primeiramente é formado o Lagrangeano de (6), que, após um manuseio matemático, é expresso por

$$
L\left(\lambda_{i}, \mathbf{w}_{i}\right)=\sum_{i=1}^{M} \lambda_{i} \sigma^{2}+\sum_{i=1}^{M} \mathbf{w}_{i}^{H}\left(I-\frac{\lambda_{i}}{\gamma_{i}} \mathbf{s}_{i} \mathbf{s}_{i}^{H}+\sum_{j \neq i}^{M} \lambda_{j} \mathbf{s}_{j} \mathbf{s}_{j}^{H}\right) \mathbf{w}_{i}
$$

onde $\lambda_{i}$, com $0<\lambda_{i}$, é o multiplicador de Lagrange para a restrição de desigualdade. Assim, se obtém a seguinte função objetivo dual:

$$
q\left(\lambda_{i}\right)=\min _{\mathbf{w}_{i}}\left[L\left(\lambda_{i}, \mathbf{w}_{i}\right)\right]
$$

$\mathrm{O}$ problema dual consiste na maximização da função objetivo $q\left(\lambda_{i}\right)$. Como não há restrições para $\mathbf{w}_{i}$, torna-se claro que $q\left(\lambda_{i}\right)=-\infty$ se o termo $I-\frac{\lambda_{i}}{\gamma_{i}} \mathbf{s}_{i} \mathbf{s}_{i}^{H}+\sum_{j \neq i}^{M} \lambda_{j} \mathbf{s}_{j} \mathbf{s}_{j}^{H}$ não for positivo semi-definido. Como $\lambda_{i}$ deve ser escolhido de forma que a função $q\left(\lambda_{i}\right)$ seja finita, a desigualdade mencionada impõe uma restrição em $q\left(\lambda_{i}\right)$ e o problema dual pode ser representado como $[9,10]$ 


$$
\begin{gathered}
\max _{\lambda_{i}} \sum_{i=1}^{M} \lambda_{i} \sigma^{2} \\
\text { sujeito a: } I+\sum_{j=i}^{M} \lambda_{j} \mathbf{s}_{j} \mathbf{s}_{j}^{H} \geq\left(1+\frac{1}{\gamma_{i}}\right) \lambda_{i} \mathbf{s}_{i} \mathbf{s}_{i}^{H} .
\end{gathered}
$$

Por outro lado, utilizando o filtro de Wiener [11], temos que

$$
\hat{\mathbf{w}}_{i}=\left(\sum_{j=1}^{M} \lambda_{j} \sigma^{2} \mathbf{s}_{j} \mathbf{s}_{j}^{H}+\sigma^{2} I\right)^{\dagger} \mathbf{s}_{i}
$$

onde $(\cdot)^{\dagger}$ representa a operação de pseudo-inversa, e considerando $\lambda_{i} \sigma^{2}=P_{i}$ como a potência dual irradiada no enlace de subida (uplink), o problema (7) pode ser reescrito como

$$
\begin{gathered}
\min _{\lambda_{i}}\left(\sum_{i=1}^{M} \lambda_{i} \sigma^{2}\right) \\
\text { sujeito a: } I+\sum_{j=i}^{M} \lambda_{j} \mathbf{s}_{j} \mathbf{s}_{j}^{H} \leq\left(1+\frac{1}{\gamma_{i}}\right) \lambda_{i} \mathbf{s}_{i} \mathbf{s}_{i}^{H},
\end{gathered}
$$

onde, para chegar a (12), são feitas algumas manipulações algébricas e utilizado o Lemma 1 proposto em [6].

Como na solução ótima a restrição de SINR deve ser ativa [10], o problema de minimização em (12) pode ser convertido em um problema de maximização com a inversão do sinal de desigualdade, chegando desta maneira, exatamente na mesma expressão do problema dual do enlace de descida (downlink), Eq. (10). Desta maneira, através da Teoria da Dualidade é comprovado que o problema do enlace de descida (downlink) pode ser resolvido através do enlace de subida (uplink), desde que não exista o chamado gap de Dualidade $[9,10]$. O gap de dualidade é o vazio que pode existir em alguns casos entre o mínimo do problema primal e o máximo do problema dual. Ou seja, as soluções dos dois problemas não coincidem. Em aplicação de arranjos de antenas, esta condição não acontece pela proximidade dos canais de subida e descida.

\section{Algoritmo de OtIMIZAÇão}

O algoritmo descrito a seguir foi proposto em [8] e [7]. Também é importante mencionar que a normalização da potência do enlace de subida (uplink) proposto em [8], faz com que se encontre uma solução ótima verdadeira. Basicamente, a normalização é realizada em relação ao ruído na forma de $\tilde{\mathbf{s}}_{i}=\mathbf{s}_{i} / \sigma_{i}$. Assim, o algoritmo é calculado iterativamente conforme a seguir:

1. Calcular os canais normalizados $\tilde{\mathbf{s}}_{i}=\mathbf{s}_{i} / \sigma_{i} \quad \forall i$

2. Aplicar o algoritmo em [7] ao problema normalizado como segue:

\section{Passo 1: Para $1 \leq i \leq M$}

$$
\begin{gathered}
\hat{\mathbf{w}}_{i}^{n}=\arg \min _{\mathbf{w}_{i}} \sum_{j \neq i}^{M} P_{j}^{n}\left|\mathbf{w}_{i}^{H} \tilde{\mathbf{s}}_{j}\right|^{2}+\left\|\mathbf{w}_{i}\right\|^{2} \\
\text { sujeito a: } \mathbf{w}_{i}^{H} \tilde{\mathbf{s}}_{i}=1
\end{gathered}
$$

Passo 2: Para $1 \leq i \leq M$

$$
P_{i}^{n+1}=\gamma_{i} \sum_{j \neq i}^{M} P_{j}^{n}\left|\left(\hat{\mathbf{w}}_{i}^{n}\right)^{H} \tilde{\mathbf{s}}_{j}\right|^{2}+\gamma_{i}\left\|\hat{\mathbf{w}}_{i}^{n}\right\|^{2}
$$

Passo 3: Para $1 \leq i \leq M$

$$
\tilde{P}_{i}^{n+1}=\gamma_{i} \sum_{j \neq i}^{M} \tilde{P}_{j}^{n}\left|\left(\hat{\mathbf{w}}_{j}^{n}\right)^{H} \tilde{\mathbf{s}}_{i}\right|^{2}+\gamma_{i}
$$

onde $\tilde{P}_{i}$ é a potência utilizada para obtenção dos pesos do enlace de descida (downlink).

3. Ao fim do algoritmo, o vetor de pesos para conformar o feixe do enlace de descida, $\mathbf{w}_{i}$, é atualizado na forma de $\mathbf{w}_{i}=\sqrt{\tilde{P}_{i}^{n f}} \hat{\mathbf{w}}_{i}^{n f}$, sendo que $n f$ é a ultima iteração do algoritmo.

A seguir, é realizada a modificação proposta neste trabalho.

\section{A. Algoritmo proposto: LMS Restrito}

Para uma implementação eficiente do Passo 1 no algoritmo anterior, propõe se neste artigo a utilização do algoritmo LMS restrito para o cálculo de $\hat{\mathbf{w}}_{i}^{n}$, através da minimização proposta em (13). O desenvolvimento do processo iterativo, aplicado ao processo dado em [12], para tal primeiramente é formulado o Lagrangeano da Eq. (13), como segue:

$$
L_{i}=\sum_{j \neq i}^{M} P_{j}^{n}\left|\mathbf{w}_{i}^{H} \tilde{\mathbf{s}}_{j}\right|^{2}+\left\|\mathbf{w}_{i}\right\|^{2}+\lambda_{i}\left(\mathbf{w}_{i}^{H} \tilde{\mathbf{s}}_{i}-1\right)
$$

sendo que $\lambda_{i}$ é o multiplicador de Lagrange para o canal $i$. Tomando o gradiente do Lagrangeano em função de $\mathbf{w}_{i}$, tem-se

$$
\nabla_{\mathbf{w}_{i}}\left(L_{i}\right)=\frac{\partial L_{i}}{\partial \mathbf{w}_{i}}=\sum_{j \neq i}^{M} P_{j}^{n} \tilde{\mathbf{s}}_{j} \tilde{\mathbf{s}}_{j}^{H} \mathbf{w}_{i}+\mathbf{w}_{i}+\frac{\lambda_{i}}{2} \tilde{\mathbf{s}}_{i}
$$

Fazendo o uso do algoritmo LMS, é construído o processo iterativo para o calculo de $\hat{\mathbf{w}}_{i}$ ótimo da seguinte forma:

$$
\hat{\mathbf{w}}_{i}(t+1)=\hat{\mathbf{w}}_{i}(t)-\mu \nabla_{\hat{\mathbf{w}}_{i}}\left\{L_{i}\left[\hat{\mathbf{w}}_{i}(t), \lambda_{i}(t)\right]\right\},
$$

onde $t$ é a iteração, $\mu$ é o passo de adaptação e $\hat{\mathbf{w}}_{i}(0)=\mathbf{w}_{i}$. Note que em (13), o processo iterativo é iniciado com o vetor de pesos do enlace de descida. Agora, substituindo (17) em (18) e pré multiplicando a equação resultante por $\tilde{\mathbf{s}}_{i}^{H}$ chega-se a

$\tilde{\mathbf{s}}_{i}^{H} \hat{\mathbf{w}}_{i}(t+1)=\tilde{\mathbf{s}}_{i}^{H} \hat{\mathbf{w}}_{i}(t)-\tilde{\mathbf{s}}_{i}^{H} \mu\left[\sum_{j \neq i}^{M} P_{j}^{n} \tilde{\mathbf{s}}_{j} \tilde{\mathbf{s}}_{j}^{H} \hat{\mathbf{w}}_{i}(t)+\hat{\mathbf{w}}_{i}(t)+\frac{\lambda_{i}(t)}{2} \tilde{\mathbf{s}}_{i}\right]$. 
A restrição (13) implica $\hat{\mathbf{w}}_{i}^{H}(t+1) \tilde{\mathbf{s}}_{i}=\tilde{\mathbf{s}}_{i}^{H} \hat{\mathbf{w}}_{i}(t+1)=1 \mathrm{e}$, dessa forma, a iteração atual caminha em direção à minimização do Lagrangeano, corrigindo a potência na direção da fonte. Dessa forma é atendida a restrição a cada iteração e encontrada uma expressão para $\lambda_{i}(n)$,

$$
\frac{\lambda_{i}(t)}{2}=-\frac{1}{\left\|\tilde{\mathbf{s}}_{i}\right\|^{2}}\left[\frac{1-\tilde{\mathbf{s}}_{i} \hat{\mathbf{w}}_{i}(t)}{\mu}+\tilde{\mathbf{s}}_{i}^{H} \hat{\mathbf{w}}_{i}(t)+\sum_{j \neq i}^{M} P_{j}^{n} \tilde{\mathbf{s}}_{i}^{H} \tilde{\mathbf{s}}_{j} \tilde{\mathbf{s}}_{j}^{H} \hat{\mathbf{w}}_{i}(t)\right] .
$$

Substituindo (20) em (18) e utilizando as relações $\tilde{\mathbf{s}}_{i}^{H} \hat{\mathbf{w}}_{i}(t) \tilde{\mathbf{s}}_{i}=\tilde{\mathbf{s}}_{i} \tilde{\mathbf{s}}_{i}^{H} \hat{\mathbf{w}}_{i}(t)$ e $\tilde{\mathbf{s}}_{i}^{H} \tilde{\mathbf{s}}_{j} \tilde{\mathbf{s}}_{j}^{H} \hat{\mathbf{w}}_{i}(t) \tilde{\mathbf{s}}_{i}=\tilde{\mathbf{s}}_{i} \tilde{\mathbf{s}}_{i}^{H} \tilde{\mathbf{s}}_{j} \tilde{\mathbf{s}}_{j}^{H} \hat{\mathbf{w}}_{i}(t)$, as seguintes expressões como solução do processo iterativo dado:

$$
\hat{\mathbf{w}}_{i}(t+1)=\mathbf{P}\left\{\hat{\mathbf{w}}_{i}(t)-\mu\left[\hat{\mathbf{w}}_{i}(t)+\sum_{j \neq i}^{M} P_{j}^{n} \tilde{\mathbf{s}}_{j} \tilde{\mathbf{s}}_{j}^{H} \hat{\mathbf{w}}_{i}(t)\right]\right\}+\frac{\tilde{\mathbf{s}}_{i}}{\left\|\tilde{\mathbf{s}}_{i}\right\|^{2}}
$$

com

$$
\mathbf{P}=\mathbf{I}-\frac{\tilde{\mathbf{s}}_{i} \tilde{\mathbf{s}}_{i}^{H}}{\left\|\tilde{\mathbf{s}}_{i}\right\|^{2}} \text { e } \hat{\mathbf{w}}_{i}(0)=\mathbf{w}_{i}=\frac{\tilde{\mathbf{s}}_{i}}{\left\|\tilde{\mathbf{s}}_{i}\right\|^{2}}
$$

A matriz $\mathbf{P}$ na Eq. (21) representa uma matriz de projeção. Como argumentado em [12], esta matriz projeta a atualização do vetor em um hiperplano de restrição nula, de forma que $\hat{\mathbf{w}}_{i}^{H} \mathbf{s}_{i}=0$, e em seguida é somado com o vetor $\tilde{\mathbf{s}}_{i} /\left\|\tilde{\mathbf{s}}_{i}\right\|^{2}$ para então atender a restrição $\hat{\mathbf{w}}_{i}^{H} \tilde{\mathbf{s}}_{i}=1$. Esta técnica corrige erros de arredondamento numérico acumulados ao longo do tempo, garantindo que a restrição a cada iteração é satisfeita. Isto pode ser verificado pré-multiplicando (21) por $\tilde{\mathbf{s}}_{i}^{H}$. Uma interpretação geométrica deste processo é ilustrada na Fig. 2.

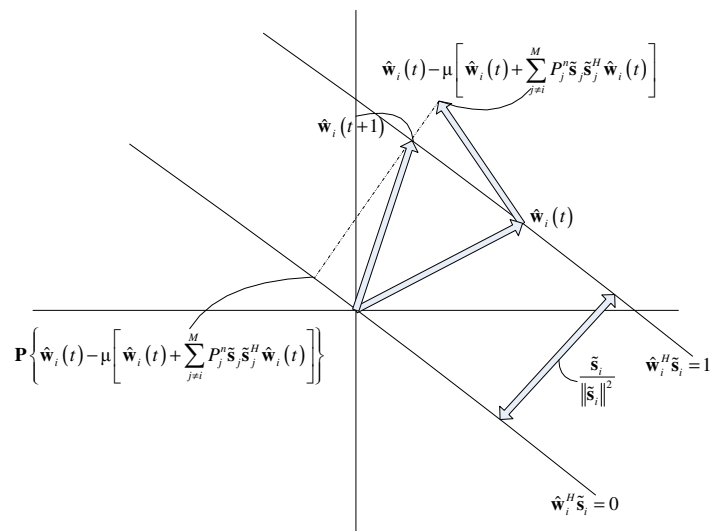

Fig. 2 - Interpretação Geométrica do Algoritmo Proposto.

\section{Resultados NumÉRICOS}

Para fins de ilustrar o método, e sem perda de generalidade, em todas as simulações é considerado um sistema MISO. O arranjo de antenas da ERB é um arranjo linear de $k$ antenas, afastadas uniformemente, com espaçamento de meio comprimento de onda da freqüência de trabalho do arranjo. $\mathrm{O}$ vetor de pesos do enlace de descida (downlink) é de $\tilde{\mathbf{s}}_{i} /\left\|\tilde{\mathbf{s}}_{i}\right\|^{2}$, e as fontes dos terminas móveis são consideradas com um ângulo de chegada de $\phi$ graus em relação à linha do eixo do conjunto. Nas simulações é considerado um ambiente livre de distorções e propagação multipercurso, sendo considerado apenas o ruído gaussiano de cada canal.

$\mathrm{O}$ passo $\mu$ foi escolhido empiricamente, seguindo os argumentos apresentados em [12]. O critério de parada das iterações em $n$ é a igualdade da $\operatorname{SINR}_{i}$ do enlace de subida (uplink), com $\gamma_{i}$ para todo $i$. A restrição de SINR $\gamma_{i}$ é definida pelo projetista do sistema de forma a atender determinados critérios. Para o término das iterações é tomado como referência a diferença absoluta da função custo (13) entre iterações consecutivas e comparado com um escalar. Tal critério é definido como

$$
\begin{aligned}
\operatorname{abs}\left(\sum_{j \neq i}^{M} P_{j}^{n}\right. & \left|\hat{\mathbf{w}}_{i}^{H}(t+1) \tilde{\mathbf{s}}_{j}\right|^{2}+\left\|\hat{\mathbf{w}}_{i}(t+1)\right\|^{2} \\
& \left.-\sum_{j \neq i}^{M} P_{j}^{n}\left|\hat{\mathbf{w}}_{i}^{H}(t) \tilde{\mathbf{s}}_{j}\right|^{2}+\left\|\hat{\mathbf{w}}_{i}(t)\right\|^{2}\right) \leq \varepsilon,
\end{aligned}
$$

onde $\varepsilon$ é um escalar positivo e suficientemente pequeno de forma a garantir um ponto próximo ao mínimo. Em todas as simulações, foi utilizado $\varepsilon=0,0001$. É importante mencionar que o enlace de descida é efetivamente adquirido somente ao fim de todo processo iterativo.

Exemplo 1: O primeiro exemplo é com cenário com 3 terminais móveis com potência inicial unitária e ângulo de chegada de $90^{\circ}, 85^{\circ}$ e $80^{\circ}$, respectivamente. A ERB possui um arranjo de 6 elementos e restrição $\gamma_{i}$ unitária para todo $i$, e ainda foi considerado o ruído dos canais com mesma variância de 0,1 . O resultado obtido com um passo de $\mu=0,01$ foi de 18 iterações em $n$ e uma média de 54 iterações para $t$, o que resulta em um total próximo de $18 \times 54$ iterações até a convergência do algoritmo. Nas Fig. 3, pode-se visualizar o diagrama de irradiação em ambos os canais para cada móvel e, na Fig. 4, é mostrado em uma dada iteração de $n$ os valores de $\hat{\mathbf{w}}_{i}^{H}(t) \tilde{\mathbf{s}}_{i}$ para determinado móvel e todo $t$. É comprovado nesta última figura a submissão à restrição para toda iteração, conforme mencionado anteriormente. As potências de transmissão $P_{i}$, determinadas para cada móvel (na última iteração) são: 0,0563; 0,1043 e 0,0568 . 

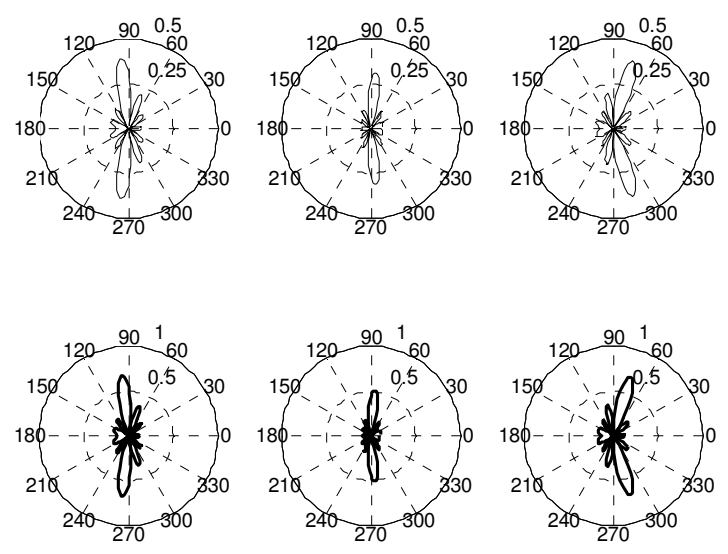

Uplink - Downlink

Fig. 3 - Diagrama de Irradiação para cada móvel da primeira simulação.

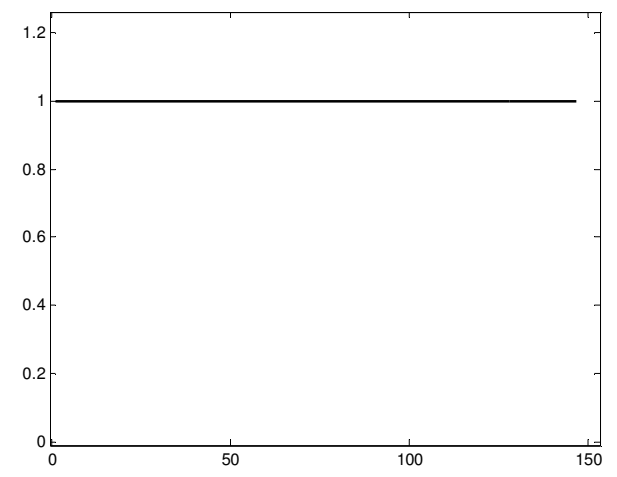

Fig. 4 - valores de $\hat{\mathbf{w}}_{i}^{H}(t) \tilde{\mathbf{s}}_{i}$ para determinado móvel.

Exemplo 2: Neste exemplo é realizada uma simulação com as mesmas características de ruído e $\gamma_{i}$ que o caso anterior. Um arranjo de 4 elementos, duas fontes com potência inicial de 10 e 1 , respectivamente, e com ângulo de chegada de $0^{\circ} \mathrm{e}$ $2^{\circ}$ é analisada. Este representa um caso um pouco mais difícil, pela proximidade das duas fontes e pelo baixo número de elementos. O número de iterações, com o mesmo $\mu$ do caso anterior, foi de $2455 \mathrm{em} n$ e de 90 em $t$, resultando em um total de $2455 \times 90$ iterações. Na Fig. 5 é ilustrado o diagrama de irradiação deste caso e, na Fig. 6, é mostrado o valor absoluto da função custo (13) para um determinado terminal móvel em uma iteração $n$ para todo $t$. Observa-se nesta última, que o passo escolhido está em uma região donde algoritmo ainda converge. Portanto, mesmo nesta condição, o esquema proposto realiza a tarefa com sucesso. As potências de transmissão $P_{i}$, para cada móvel, foram (ao fim da aplicação do algoritmo proposto) 12,7460 e 12,7472 para o móvel 1 e 2, respectivamente. Note que foram achados valores maiores que no caso anterior justamente pela maior dificuldade em atingir a restrição de SINR dada.
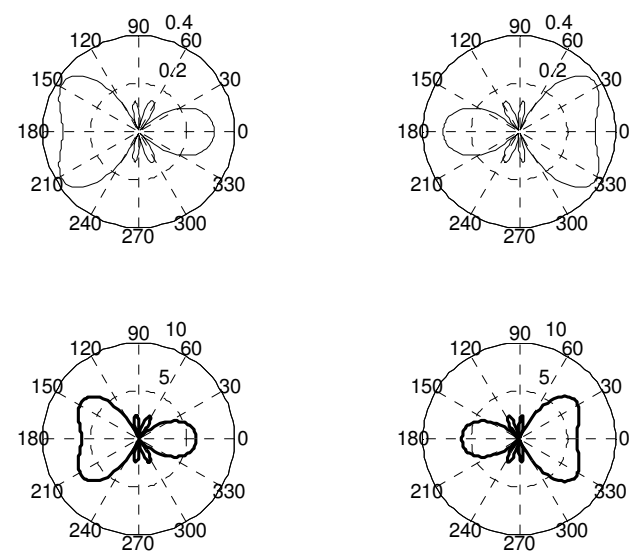

- Uplink $\longrightarrow$ Downlink

Fig. 5 - Diagrama de Irradiação para cada móvel da segunda simulação.

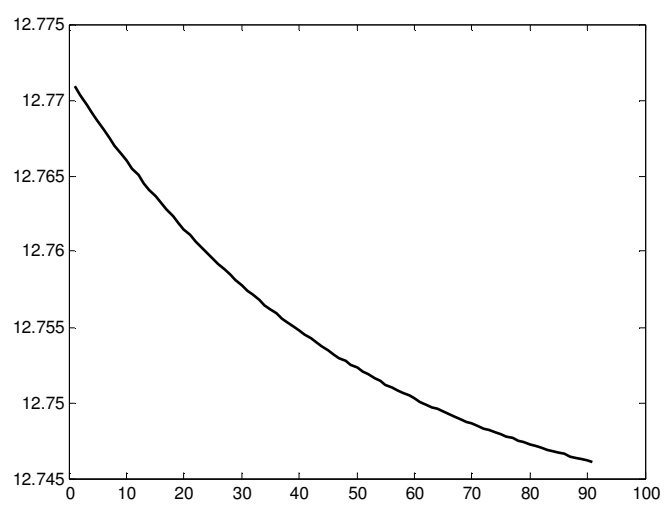

Fig. 6 - Gráfico da função custo $\sum_{j \neq i}^{M} P_{j}^{n}\left|\mathbf{w}_{i}^{H}(t) \tilde{\mathbf{s}}_{j}\right|^{2}+\left\|\mathbf{w}_{i}(t)\right\|^{2}$ em uma determinada iteração $n$.

Exemplo 3: Este exemplo é utilizado para ilustrar a convergência da curva de SINR para ambos os enlaces dos usuários. O arranjo utilizado foi de 6 elementos, variância do ruído de 0,5, 2 usuários móveis posicionados com ângulos de chegada de $90^{\circ}$ e $89^{\circ}$, potência inicial de 2 e 5 e restrições de SINR de 1 e 2, respectivamente. A Fig. 7 mostra a curva de convergência da SINR para ambos os móveis em cada canal. Neste caso foi adquirido o vetor de pesos do canal de enlace de descida durante o processo iterativo, verificando a convergência da curva de SINR deste canal. O passo utilizado para este caso foi o mesmo que nos exemplos anteriores. 


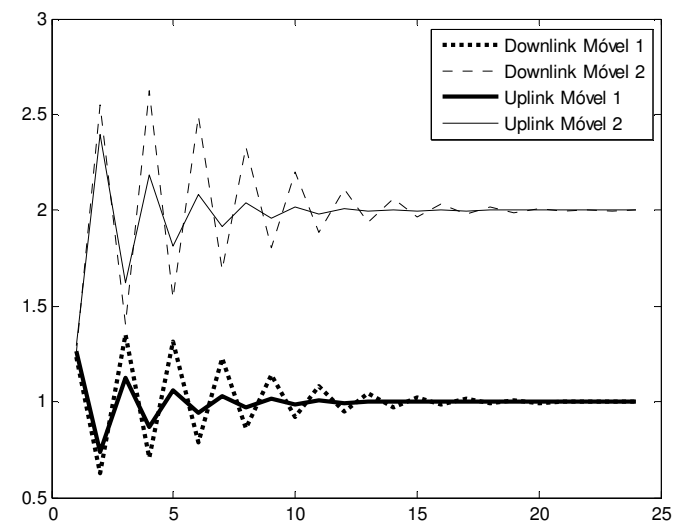

Fig. 7 - Convergência da SINR para a terceira simulação.

\section{CONCLUSÕES}

Neste trabalho, foi analisada pela Teoria de Dualidade a relação entre os problemas dos enlaces de subida e descida. Foi então inserido no algoritmo proposto em [8], uma modificação aqui proposta, o algoritmo LMS restrito, para a resolução do problema de otimização apresentado. O algoritmo resultante une a conformação de feixe dos enlaces de subida e descida (uplink e downlink) e converge para a igualdade da restrição de SINR com a menor potência total possível. O algoritmo proposto é computacionalmente simples, pois não requer nenhuma operação de maior dificuldade como a inversão de matrizes como o caso de outros algoritmos (RLS, APA, etc.).

\section{REFERÊNCIAS}

[1] L. B. Fertig and J. H. McClellan, "A dual form adaptive filter," in Acoustics, Speech, and Signal Processing, 1990. ICASSP-90., 1990 International Conference on, 1990, pp. 1433-1436 vol.3.

[2] L. B. Fertig and J. H. McClellan, "Dual forms for constrained adaptive filtering," Signal Processing, IEEE Transactions on, vol. 42, pp. 11-23, 1994.

[3] P. I. Ioannides and C. A. Balanis, Introduction to Smart Antennas: Morgan \& Claypool, 2007.

[4] W. Yu and T. Lan, "Downlink beamforming with per-antenna power constraints," in Signal Processing Advances in Wireless Communications, 2005 IEEE 6th Workshop on, 2005, pp. 1058-1062.

[5] M. Codreanu, A. Tolli, M. Juntti, and M. Latva-aho, "Uplink-Downlink SINR Duality via Lagrange Duality," in Wireless Communications and Networking Conference, 2008. WCNC 2008. IEEE, 2008, pp. 1160-1165.

[6] Y. Wei and T. Lan, "Transmitter Optimization for the Multi-Antenna Downlink With Per-Antenna Power Constraints," Signal Processing, IEEE Transactions on, vol. 55, pp. 2646-2660, 2007.

[7] F. Rashid-Farrokhi, K. J. R. Liu, and L. Tassiulas, "Transmit beamforming and power control for cellular wireless systems," Selected Areas in Communications, IEEE Journal on, vol. 16, pp. 1437-1450, 1998.

[8] E. Visotsky and U. Madhow, "Optimum beamforming using transmit antenna arrays," in Vehicular Technology Conference, 1999 IEEE 49th, 1999, pp. 851-856 vol.1.

[9] S. Boyd and L. Vandenberghe, Convex Optimization. Los Angeles: cambridge university press, 2008.

[10] D. P. Bertsekas, Nonlinear Programming, Second ed. Belmont: Athena Scientific, 1999.

[11] L. C. Godara, Smart Antennas. Boca Raton, London, New York, Washington, D.C: CRC PRESS, 2004.

[12] O. L. Frost, III, "An algorithm for linearly constrained adaptive array processing," Proceedings of the IEEE, vol. 60, pp. 926-935, 1972. 